\title{
Apgar Score at Ten Minutes
}

National Cancer Institute

\section{Source}

National Cancer Institute. Apgar Score at Ten Minutes. NCI Thesaurus. Code C98817.

The numerical sum between zero and ten that is computed from individual values of zero to two that are assigned to five different areas of interest in newborn screenings at ten minutes after birth. These areas are breathing, muscle tone, pulse rate, reflex irritability and skin color. 